Résumés des conférences et travaux

\title{
Sudarabique et ouest sémitique
}

\section{François Bron}

\section{OpenEdition \\ Journals}

Édition électronique

URL : https://journals.openedition.org/ashp/633

DOI : 10.4000/ashp.633

ISSN : 1969-6310

Éditeur

Publications de l'École Pratique des Hautes Études

\section{Édition imprimée}

Date de publication : 1 octobre 2009

Pagination : 33-35

ISSN : 0766-0677

\section{Référence électronique}

François Bron, "Sudarabique et ouest sémitique ", Annuaire de l'École pratique des hautes études

(EPHE), Section des sciences historiques et philologiques [En ligne], 140 | 2009, mis en ligne le 05 octobre 2009, consulté le 06 juillet 2021. URL : http://journals.openedition.org/ashp/633 ; DOI : https://doi.org/ 10.4000/ashp.633 


\title{
SUDARABIQUE ET OUEST SÉMITIQUE
}

\author{
Directeur d'études : M. François Bron
}

Programme de l'année 2007-2008 : I. Inscriptions puniques. - II. Le royaume de Saba au III ${ }^{e}$ siècle de notre ère.

\section{Inscriptions puniques}

En épigraphie punique, une bonne partie de l'année a été consacrée à une étude approfondie des tarifs sacrificiels. On a commencé par le tarif dit « de Marseille » (CIS 165) - quoique sa provenance de Carthage ne fasse guère de doute - qui est le mieux conservé. On est passé ensuite aux différents fragments trouvés à Carthage même : CIS 167 et CIS 3915, 3916 et 3917, qui semblent appartenir à plusieurs exemplaires d'un même tarif, légèrement différent de celui de Marseille et beaucoup moins bien préservé. On s'est heurté aux mêmes difficultés que nos devanciers concernant le vocabulaire de ces textes : les termes désignant les différentes catégories de sacrifices restent très obscurs. Il en va de même pour ceux qui nomment les diverses parties du corps des victimes attribuées aux participants et même pour les noms de certains des animaux sacrifiés. On s'est penché enfin sur les parallèles qu'on a voulu instituer entre les prescriptions de ces tarifs et les sacrifices décrits dans les chapitres 1 à 7 du Lévitique. Si la parenté des deux catégories de textes est évidente et reflète un fond commun de civilisation, il est illusoire de vouloir instituer un parallèle terme à terme entre les divers types de sacrifices mentionnés, comme l'avait fait R. Dussaud il y a maintenant quatre-vingts ans. Mais il est vrai que des études plus récentes, tout en revenant périodiquement sur la question, se font nettement plus prudentes.

On a revu ensuite le petit fragment CIS 166, qui contient les restes de deux colonnes de texte : il devait s'agir d'une sorte de calendrier rituel des sacrifices, analogue aux textes de ce genre découverts à Ougarit. Seules nous sont conservées en partie les prescriptions concernant les quatrième et cinquième jours d'un mois inconnu. On a pu constater que ce texte a été découvert en 1872, que les derniers fragments de tarifs sacrificiels l'ont été vers 1920 et que depuis, malgré une activité archéologique intense sur le site de Carthage, plus aucun texte de ce genre n'a été retrouvé.

On a réétudié également l'unique exemple de tabella devotionis punique, CIS 6068, dont toutes les difficultés ne sont pas encore résolues, malgré les nombreuses études qui lui ont été consacrées.

Enfin, en dernière heure, on a revu, grâce aux estampages conservés au cabinet du Corpus, plusieurs inscriptions funéraires néo-puniques, celles de Kef Bezioun et une autre, provenant du djebel Guelala, près de Souk Ahras, publiée par Allotte de la Füye dans le Recueil des notices et mémoires de la Société archéologique de Constantine, vol. 24, paru en 1888, et qui a disparu depuis lors de la littérature scientifique.

\section{Sudarabique}

En sudarabique, la majeure partie de l'année a été consacrée à l'étude des nouvelles inscriptions provenant du pillage des sites du Jawf, publiées par M. Arbach et 
R. Audouin, San 'â' National Museum - Collection of Epigraphic and Archaeological Artifacts from al-Jawf Sites - Part II, Sanaa, 2007. Ces textes, dont beaucoup remontent à la première moitié du premier millénaire avant notre ère, sont une mine de renseignements sur la période la plus ancienne de la civilisation sudarabique, lorsque le Jawf était encore constitué d'une mosaïque de petites cités-États indépendantes. On a corrigé au passage les nombreuses erreurs typographiques, qui obligent à consulter à la fois la photographie et les diverses transcriptions, en caractères sudarabiques, latins et arabes, pour s'assurer du texte.

Parmi les nouvelles inscriptions d'Inabba', YM 29828 nous fait connaître pour la première fois le symbole du dieu Samî', une sorte de panthère ou de griffon. Dans YM 29830, il est facile de compléter le nom de l'auteur de l'inscription, $H]$ ' $n$ ' $\underline{t}$. Dans YM 29831, on peut restituer, aux 1. 5-6, la formule si fréquente dans les inscriptions du Jawf, $k r b$ tkrb $b n$ - $h w$, « offrande qu'a vouée son fils ».

Les inscriptions de Jidfir ibn Munayhir sont presque toutes des dédicaces de personnes provenant d'un temple d'Almaqah qui n'avait pas été pillé jusqu'à maintenant. Parmi elles, YM 18352 revendique l'acquisition d'une palmeraie et de son canal d'irrigation. Dans YM 18350, la mention de 't $l m$, traduit habituellement par « tamaris », ne semble pas convenir au contexte; il pourrait s'agir plutôt d'un terme d'irrigation.

En ce qui concerne les textes de Haram, YM 28975 nous apprend que le dieu 'Athtar Ba'sān, connu uniquement sur ce site, est en réalité une déesse, ce qui explique sa place à la fin des invocations aux divinités. On a revu à cette occasion une autre dédicace à cette déesse, gravée sur une table à libations dont la photographie a été publiée dans un catalogue de vente de Christie's New York, en date du 8 juin 2005, $\mathrm{n}^{\mathrm{o}} 53$.

Parmi les monuments provenant d'al-Bayḍā', une brève étude est consacrée à la statue de bronze restaurée dans les laboratoires du musée du Louvre. On regrettera que le méritoire déchiffrement de ce texte difficilement lisible et sa traduction ne soient pas plus explicitement attribués à leur auteur, Iwona Gajda.

Le site d'as-Sawdā' est devenu fameux depuis le pillage du temple du dieu local Aranyada', qui a mis au jour une série de piliers, gravés de scènes représentant les principales divinités des cités du Jawf. M. Arbach publie trois inscriptions provenant de ce temple; parmi elles, YM 11191 a déjà été étudiée par A. Avanzini, Studi sul Vicino Oriente antico dedicati alla memoria di Luigi Cagni, Naples, 2000, vol. III, p. 1231-1247. Dans YM 28491, autre dédicace à Aranyada', la fin de l'énumération des dédicants, à la 1. 3, peut se lire comme suit : $w$-Msr't 'ršw 'rnyd'-šym- 'ššn, «... et Msr't, prêtres de Aranyada', patron des Nashshānites ». D'autres textes proviennent du temple hors les murs de 'Athtar, fouillé par J.-F. Breton, sans que cela soit toujours indiqué : c'est le cas de YM 28489, qui correspond à SW-BA/I/20 (cf. J.-F. Breton, « Le sanctuaire de 'Athtar dhū-Rișaf d'as-Sawdā' (République du Yémen) », CRAI, 1992, p. 429-453). Dans ce texte, on peut restituer avec vraisemblance, aux 1 . 1-2, le patronyme $\underline{H}[l]$ ' $l y$, avec une forme archaïque du $\underline{h}$, attestée uniquement dans Kamna 18. A la 1. 3, Rhś est le nom d'un dieu local, déjà connu par as-Sawdā' 5/2. L'autel YM $16620+16621$ correspond à SW-BA 14 ; la lecture et la traduction correctes en ont été données par Chr. Robin dans la communication de J.-F. Breton 
citée plus haut, p. 447. YM 16631 correspond à SW-BA 9 . Dans YM 16710 B, ’dm Strrn désigne bien entendu les « vassaux (du clan) Sațān ».

Un certain nombre d'inscriptions minéennes proviennent soit de Ma'în, soit de Barâqish, sans qu'il soit possible de décider entre les deux sites. Deux textes importants, comptant respectivement six et sept lignes, YM 28980 et YM 28981, se révèlent d'une difficulté exceptionnelle. Ce sont des blocs provenant sans doute d'inscriptions gravées sur la paroi d'un édifice. Les textes sont donc incomplets et même le sens général reste difficile à préciser. YM 26106 est une inscription pénitentielle dédiée au dieu Wadd, à l'occasion, semble-t-il, d'une crue du « fleuve de Wadd ». Enfin YM 28488 comporte deux textes parallèles fixant les parts de sacrifices à' 'Athtar dhûQabḍ et Wadd attribuées, d'une part aux dieux de Ma'în, d'autre part, soit au roi de Ma'în, soit aux deux prêtres de Wadd.

On a présenté enfin trois antiquités inédites appartenant à la collection Moussaïeff : un autel à encens qatabanite dédié au dieu Wadd, la statuette en albâtre de la fille d'un roi de Qatabân et un gobelet en bronze sabéen, dédié par une femme au dieu Sami'. 